\title{
Non-Neutropenic Sepsis in Cancer Patients: The Luminal Obstruction Syndrome
}

\author{
Tamás Kullmann* ${ }^{*}$, Dóra Füzi, Tamás Pintér \\ Department of Oncoradiology, Petz Aladár Hospital, Győr, Hungary \\ Email: *kullmanndoki@hotmail.com
}

How to cite this paper: Kullmann, T., Füzi, D. and Pintér, T. (2019) Non-Neutropenic Sepsis in Cancer Patients: The Luminal Obstruction Syndrome. Journal of Cancer Therapy, 10, 789-795. https://doi.org/10.4236/jct.2019.1010066

Received: August 28, 2019

Accepted: September 26, 2019

Published: September 29, 2019

Copyright $\odot 2019$ by author(s) and Scientific Research Publishing Inc. This work is licensed under the Creative Commons Attribution International License (CC BY 4.0).

http://creativecommons.org/licenses/by/4.0/ (c) (i) Open Access

\begin{abstract}
Background: Neutropenic sepsis is a frequent complication of cytotoxic chemotherapy delivered for cancer patients. Its management is well determined by clinical guidelines. Non-neutropenic sepsis is another potential complication in cancer patients. Its management is less established in the medical literature. Materials and Methods: Three cases are presented to illustrate favourable evolution of non-neutropenic biliary-, uro- and bronchogenic-sepsis in cancer patients with poor prognosis. Results: All three patients had a survival over six months after the management of the septic complication. Two of them received subsequent systemic anticancer treatment. Conclusions: Survival benefit offered by the management of cancer-related non-neutropenic sepsis may be comparable to the benefit obtained by systemic anticancer treatments. Cost-effectiveness of sepsis management may be better than that of anticancer treatments.
\end{abstract}

\section{Keywords}

Sepsis, Biliary Sepsis, Urosepsis, Bronchogenic Sepsis, Solid Cancer

\section{Introduction}

Sepsis is a frequent complication of solid tumours. Three iatrogenic risk factors are generally identified, responsible of specific clinical pictures: major surgeryrelated "postoperative sepsis" [1], cytotoxic chemotherapy-related "neutropenic sepsis" [2] and central catheter-related "catheter sepsis" [3]. The management of these conditions is well described by clinical guidelines. Although de novo, non-neutropenic sepsis occurs frequently in cancer patients as well, its importance is not sufficiently discussed in the medical literature [4].

The diagnosis of sepsis is not difficult when all of the specific clinical signs are present and there are no disturbing laboratory results. However, when the only 
clinical sign is the alteration of the general status of the patient (without fever and organ-specific symptoms) and a large scale of laboratory parameters are disturbed (including renal and liver functions, as well as haemostasis), clinicians may be tempted to explain the situation with the progression of the underlying malignant disease. The establishment of the diagnosis and the initiation of antibiotic treatment may suffer considerable delay, thus increasing mortality.

The mortality rate of sever sepsis correlates inversely with the interval to start empiric antibiotic treatment and if necessary, intensive care [5] [6]. Invasive interventions such as biliary or urinary deviation may be needed in addition to efficacious empiric and targeted antibiotic treatment for the successful control of the infectious complication.

The most frequent sites of sepsis are the biliary, the urinary and the respiratory tracts [7]. Three cases are presented in order to illustrate favourable evolution of cholangio-, uro-, and bronchogenic-sepsis in cancer patients with poor prognosis. All of the three patients gave their written consent for the publication of their medical history.

\section{Patients and Methods}

Case 1: A 70-year-old man was diagnosed with Klatskin tumour upon the appearance of jaundice without abdominal pain. Explorative laparotomy found the situation to be irresectable. A biliary metal stent was inserted by percutaneous approach. Three weeks later he was readmitted to hospital with fever, hypotension and loss of consciousness, corresponding to the clinical tableau of septic shock. Empiric antibiotic treatment by ceftriaxone and methronidazole and fluid resuscitation were immediately started. Three days later a subcutaneous abscess appeared in the right upper quadrant. It was punctured and thus transformed to bilio-cutaneous fistula. E. faecium was identified by culture of the purulent effusion. The patient became apyretic and was discharged at day twelve. The sequential antibiotic treatment was followed for a total of four weeks. The bilio-cutaneous fistula also closed spontaneously some weeks later.

Case 2: An 85-year-old woman was diagnosed with pelvic and pulmonary recurrence of a three years earlier operated coecum tumour upon the onset of obstructive renal insufficiency (Figure 1(a)). She had a history of left renal atrophy. The insertion of a ureteral catheter and subsequently a transrenal drain allowed the resolution of the renal pelvic dilatation and the normalisation of the renal function. She was readmitted to the department of urology for pyelonephritis associated with altered consciousness. E. Coli was identified by haemoculture. A fifteen day long targeted antibiotic treatment with cefixime allowed definitive apyrexia and complete recovery of her formal mental status.

Case 3: For a 56-year-old woman who presented with a neglected right sided breast cancer, the staging scanner showed multiple pulmonary metastases with atelectasis of the right middle and lower pulmonary lobes and consecutive right 


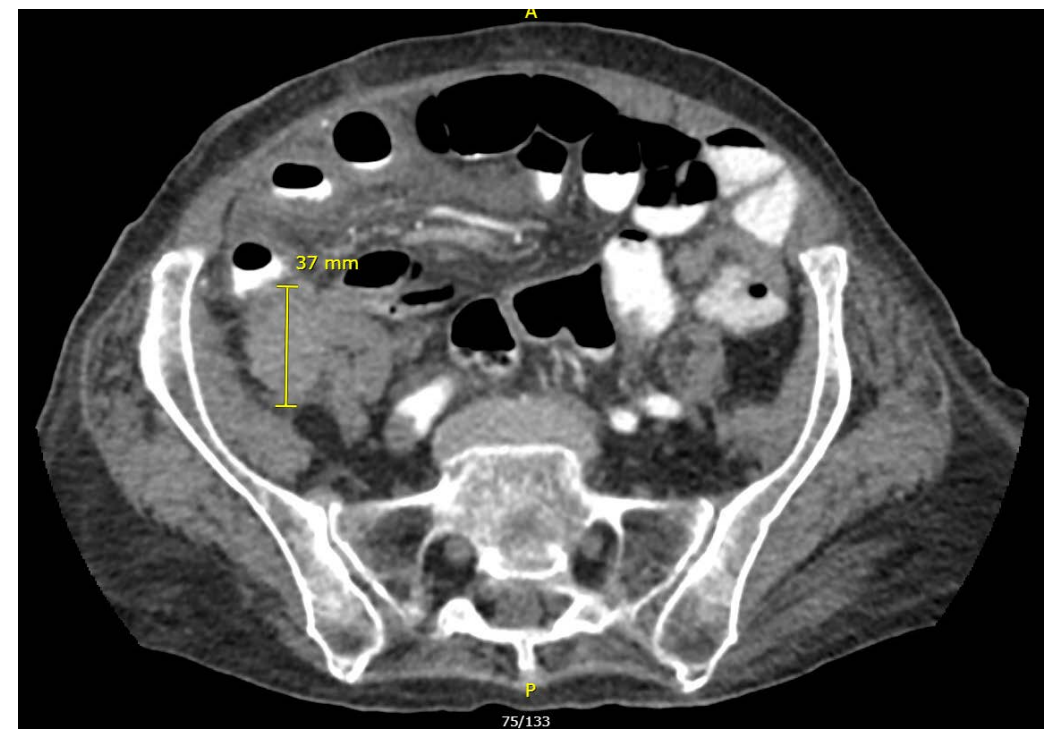

(a)

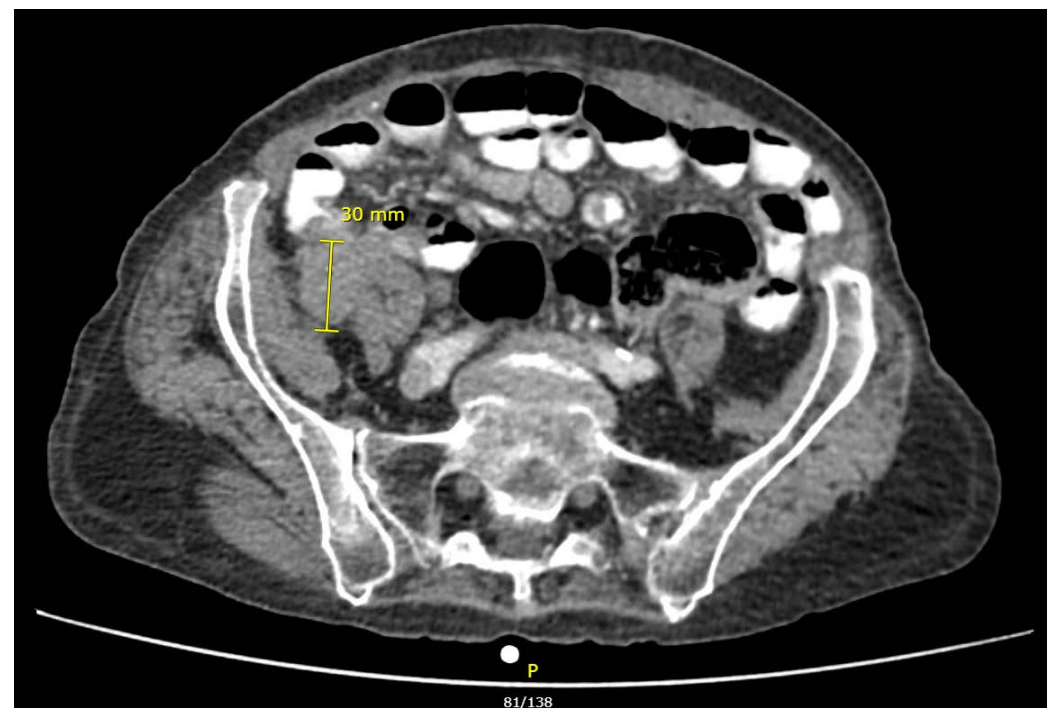

(b)

Figure 1. Partial regression of the pelvic lesion under chemotherapy.

hydrothorax (Figure 2(a)). The initiation of chemotherapy with paclitaxel allowed the regression of the carcinosis of the thoracic wall, but did not change the reproduction of the right hydrothorax that had to be punctured several times. The pleurodesis was refused by the thoracic surgeons for the presence of atelectasis. After the fifth cycle she was hospitalised with fever and dyspnoea corresponding to right sided pneumonia. Bacterial culture of the sputum was positive for $H$. parainfluenzae. Targeted antibiotic treatment with ceftriaxone allowed apyrexia. She was discharged with the prescription of prolonged sequential antibiotic treatment with cefixime. She was readmitted two more times for the same symptomatology, always some days after stopping the antibiotics, once with haemodynamic instability. The restauration of the antibiotic treatment allowed an improvement every time and it had to be maintained for more than four 


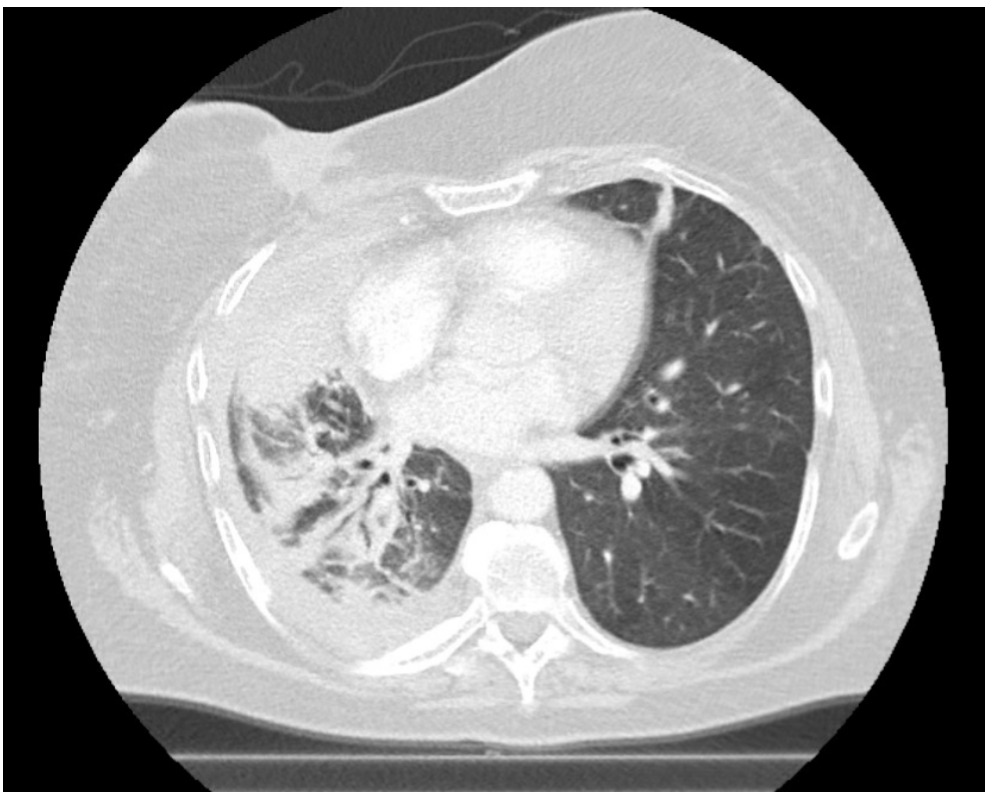

(a)

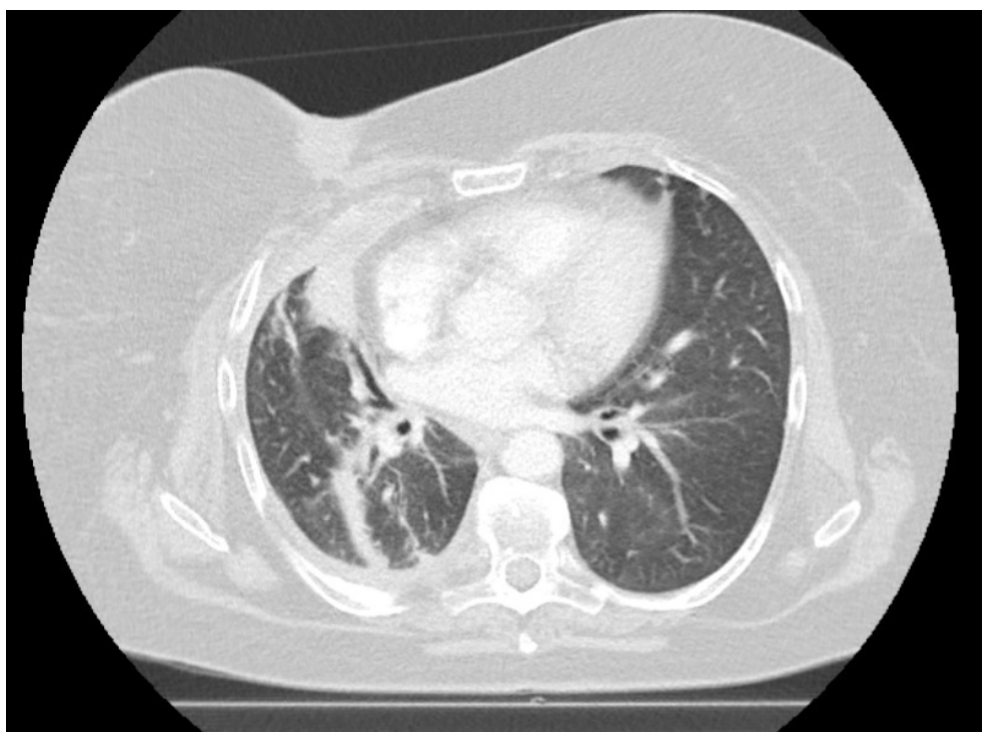

(b)

Figure 2. Decrease of the extent of atelectasis under chemotherapy.

months after the first hospitalisation, until the tenth cycle of paclitaxel. In parallel the atelectasis of the right middle and lower lobes improved substantially.

\section{Results}

Case 1: At the time of the management of this patient there was no proven efficacious chemotherapy for cholangiocellular carcinoma [8]. Taking into account the expected benefits and the potential risks he accepted not to get any anticancer treatment. He stayed completely autonomous for 8 months and was finally lost for tumour progression 10 months after the resolution of the biliary 
sepsis.

Case 2: After the control of the infectious complication palliative chemotherapy was started. It was well tolerated by the patient who has been completely autonomous despite of her high age. Control CT scan showed partial remission 3 months later (Figure $1(\mathrm{~b})$ ). She did not require any recurrent antibiotic treatment. The attempts to remove the transrenal drain have still not been successful. The treatment has been ongoing for 6 months without complication at the moment of the submission of the article.

Case 3: After improvement of the atelectasis (Figure 2(b)) the patient's oncologic treatment was changed to antihormonal therapy of which she received two lines. She also underwent choledochotomy for recurrent biliary pancreatitis. She stayed completely autonomous for more than 3 years and was finally lost for tumour progression 46 months after the resolution of the iterative pneumonia.

\section{Discussion}

Three cases of non-neutropenic sepsis were presented. The patient with Klatskin tumour related biliary sepsis had a survival of 10 months without any anticancer therapy. The patient with pelvic recurrence related urosepsis has a survival over 6 months and she is receiving ongoing palliative chemotherapy that is well tolerated. The patient with compressive pulmonary metastases related bronchogenic sepsis had a survival of 46 months with two lines of antihormonal therapy.

The cause and the physiopathology of the septic complications may be debated. There are authors who consider the primary tumour or the metastases as source of the infection [7]. We believe that the infection results of the retention of the physiologic secretion. As far as the drainage of the biliary, urinary and bronchial tracts is allowed, their bacterial contamination may not progress to infection. However, the mechanical obstruction of these luminal organs leads unavoidably to microbial overgrowth, organ-specific infection and septicaemia even in patients having an intact immune system. We like to call this phenomenon luminal obstruction syndrome. In case a patient affected with any primary or secondary tumour lesions potentially obstructing the extra- or intra-hepatic biliary tract, the ureters or the lower airways presents with an altered general status, our first clinical hypothesis is the infectious complication of the underlying obstructive malignancy. This hypothesis allows early diagnosis and treatment of organ-specific non-neutropenic sepsis.

The resolution of the obstruction is part of the management of these complications. In case of biliary obstruction, the insertion of an internal or external biliary drain, in case of urosepsis, the insertion of a ureteral catheter or a transrenal drain may ensure the biliary and urinary clearance respectively. In some cases, especially in bronchogenic sepsis, when the obstruction cannot be mechanically levied the restitution of the luminal clearance may need chemical desobstruction. In these cases, prolonged oral antibiotic treatment is also needed until the control of the infectious complication is definitely achieved. 
The presented cases had an over the average benefit of the management of the septic complication. Sever sepsis remains undoubtedly a condition with high mortality. In a recent study, $6.5 \%$ of cancer patients admitted to hospital for non-neutropenic sepsis died within 30 days [4]. Nevertheless, early diagnosis and treatment may allow a survival benefit comparable to that of the most innovative anticancer treatments. In addition, in many instances, the control of sepsis is required for the administration of anticancer treatments potentially compromising the immune defence.

The costs of a two weeks long intravenous antibiotic therapy with or without the pose of biliary or urinary catheters or drains are negligible as compared to the costs of novel anticancer agents. Thus, in addition to being efficacious sepsis management of cancer patients is cost-effective.

\section{Conclusion}

Survival benefit offered by the management of cancer-related non-neutropenic sepsis may be comparable to the benefit obtained by systemic anticancer treatments. Cost-effectiveness of sepsis management may be better than that of anticancer treatments.

\section{Conflicts of Interest}

The authors declare no conflicts of interest regarding the publication of this paper.

\section{References}

[1] Sood, A., Abdollah, F., Sammon, J.D., Arora, N., Weeks, M., Peabody, J.O., Menon, M. and Trinh, Q.D. (2017) Postoperative Sepsis Prediction in Patients Undergoing Major Cancer Surgery. Journal of Surgical Research, 209, 60-69. https://doi.org/10.1016/j.jss.2016.09.059

[2] Kochanek, M., Schalk, E., von Bergwelt-Baildon, M., Beutel, G., Buchheidt, D., Hentrich, M., Henze, L., Kiehl, M., Liebregts, T., von Lilienfeld-Toal, M., Classen, A., Mellinghoff, S., Penack, O., Piepel, C. and Böll, B. (2019) Management of Sepsis in Neutropenic Cancer Patients: 2018 Guidelines from the Infectious Diseases Working Party (AGIHO) and Intensive Care Working Party (iCHOP) of the German Society of Hematology and Medical Oncology (DGHO). Annals of Hematology, 98, 1051-1069. https://doi.org/10.1007/s00277-019-03622-0

[3] Raad, I. and Chaftari, A.M. (2014) Advances in Prevention and Management of Central Line-Associated Bloodstream Infections in Patients with Cancer. Clinical Infectious Diseases, 59, 340-343. https://doi.org/10.1093/cid/ciu670

[4] Wilson, T., Cooksley, T., Churchill, S., Radford, J. and Dark, P. (2018) Retrospective Analysis of Cancer Patients Admitted to a Tertiary Centre with Suspected Neutropenic Sepsis: Are C-Reactive Protein and Neutrophil Count Useful Prognostic Biomarkers? Journal of the Intensive Care Society, 19, 132-137. https://doi.org/10.1177/1751143717741248

[5] Ferrer, R., Martin-Loeches, I., Phillips, G., Osborn, T.M., Townsend, S., Dellinger, R.P., Artigas, A., Schorr, C. and Levy, M.M. (2014) Empiric Antibiotic Treatment Reduces Mortality in Severe Sepsis and Septic Shock from the First Hour: Results 
from a Guideline-Based Performance Improvement Program. Critical Care Medicine, 42, 1749-1755. https://doi.org/10.1097/CCM.0000000000000330

[6] de Montmollin, E., Tandjaoui-Lambiotte, Y., Legrand, M., Lambert, J., Mokart, D., Kouatchet, A., Lemiale, V., Pène, F., Bruneel, F., Vincent, F., Mayaux, J., Chevret, S. and Azoulay, E. (2013) Outcomes in Critically Ill Cancer Patients with Septic Shock of Pulmonary Origin. Shock, 39, 250-254. https://doi.org/10.1097/SHK.0b013e3182866d32

[7] Gudiol, C., Aguado, J.M. and Carratalà, J. (2016) Bloodstream Infections in Patients with Solid Tumors. Virulence, 7, 298-308.

https://doi.org/10.1080/21505594.2016.1141161

[8] Sebbagh, S., Roux, J., Dreyer, C., Neuzillet, C., de Gramont, A., Orbegoso, C., Hentic, O., Hammel, P., de Gramont, A., Raymond, E., André, T., Chibaudel, B. and Faivre, S. (2016) Efficacy of a Sequential Treatment Strategy with GEMOX-Based Followed by FOLFIRI-Based Chemotherapy in Advanced Biliary Tract Cancers. Acta Oncologica, 55, 1168-1174. https://doi.org/10.1080/0284186X.2016.1191670 